The following pages constitute the final, accepted and revised manuscript of the article.

\author{
Juan Merlo, Bo Gullberg
}

"Controlled withdrawal of selective serotonin reuptake inhibitors in elderly patients in nursing homes with no indication of depression-a commentary"

Eur J Clin Pharmacol. 2005 Jun;61(4):321-3

Publisher: Springer.

Use of alternative location to go to the published version of the article requires journal subscription.

Alternative location: http://dx.doi.org/10.1007/s00228-005-0912-y 


\title{
Controlled withdrawal of selective serotonin reuptake inhibitors in elderly patients in nursing homes with no indication of depression-a commentary
}

\author{
Eur J Clin Pharmacol (2005) 61: 321-323 DOI 10.1007/s00228-005-0912-y
}

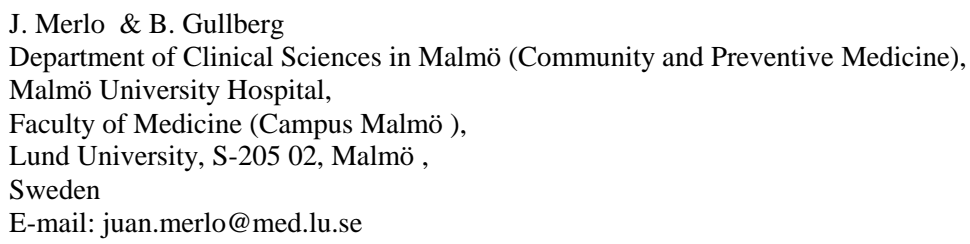

An authors’ reply to this Letter to the Editors is available at http:// dx.doi.org/10.1007/s00228-005-0915-8

\section{Introduction}

Recent investigations performed by the Swedish National Board of Health and Welfare have indicated that as many as $40 \%$ of the elderly in nursing homes are on anti-depressive drugs, mainly modern selective serotonin reuptake inhibitors (SSRIs) such as citalopram and sertraline. These studies have also shown that in many cases SSRI treatment is administered without a correct indication, and therefore one quality indicator of medicine use in the elderly $[1,2]$ is the percentage of patients on SSRI treatment without a correct indication.

Increasing the quality of medication in nursing homes is a humane, social, and medical priority and any study aimed at this goal should be encouraged. We have read with great interest an article published by Ulfvarson et al. [3]. The study—directed by Christer von Bahr—was an open, randomized, non-placebo controlled clinical trial aimed at investigating the effects of withdrawal of SSRIs in elderly nursing home patients with no documented diagnosis or symptoms of depression.

Of a total of 350 patients on SSRI treatment from 11 nursing homes in the county of Stockholm, Sweden, 70 patients aged 75 years or older with no current depressive symptoms and no history of depression or anxiety and who were taking citalopram or sertraline for at least 6 months met the inclusion criteria and consented to participate. Patients were randomized to either intervention (withdrawal of SSRI) or the control group (no change in treatment). 
According to the authors, the main outcomes were standardized measures of depression, global functioning, and health. After 6 months of follow-up, the authors found no significant difference between the intervention and control groups in any outcome measure. The authors concluded that treatment with SSRIs in patients without major clinical depression or anxiety disorder is often unjustified and should be discontinued.

The conclusion of this investigation seems to confirm the established indications for withdrawing SSRI treatment in elderly patients [1]. Unfortunately, the study contains so many flaws in both design and analysis, which make it an unacceptable source of evidence.

The authors seem to be unaware on any guidelines for good clinical trial practice or recommendation for publication of clinical trials as the CONSORT statement, which is a relevant research tool to improve the quality of reports of randomized trials [http:// www.consortstatement.org/].

In general many aspects of the trial [2] lack transparency, which makes it difficult to understand how the trial was designed, conducted, and analyzed.

\section{Possible irregularities in the randomization procedure}

Table 1 of the article indicates the existence of large differences between the control and the intervention groups after randomization. The number of women in the control group (17) is almost three times higher than in the intervention group (6). Also, the global assessment score differs.

Only 3 patients in the control group but 12 patients in the intervention group are on sertraline. The authors affirm that this last difference is not "significant"' and report a P value equal to 0.09 using the chi-square test. A simple check discovered that the $\mathrm{P}$ value reported by the authors was incorrect. The correct $\mathrm{P}$ value was in fact equal to 0.009 and therefore highly significant. Even if such a difference in the compositions of the control and intervention groups is not completely impossible after randomization, such an unbalanced result suggests irregularities in the randomization procedure - a possibility that is obscurely suggested in the article (on page 739, first paragraph). Given that the main reason for randomization is the control of known and unknown confounding factors, an unbalanced randomization further decreases the validity of this open, non-blind, non-placebo controlled trial.

It is not apparent whether randomization, in reality, was performed at the physician level (observe that each nursing home has a single responsible physician) or at the patient level. Obviously the intervention was directed at the physician level, since it was the physician who received the information and performed the SSRI withdrawal in the intervention group. If the randomization was done at the patient level and since the trial was not blind, there would be a high risk of "contamination" which may condition the physician to restart treatment in the intervention group or withdraw treatment in the control group influenced by the aims of the trial. 
It is also possible that intervention patients received substitute therapies such as other medication or special support. On this basis, it is remarkable that changes in the use of concomitant drugs were not registered.

\section{Errors in statistical power calculation and erroneous conclusions}

Power calculation - aimed to calculate the appropriate sample size for studying the primary endpoint - was carried out to investigate depressive symptoms according to the Montgomery-A sberg Depression Rating Scale (MADRS). Amazingly, the outcome used for power calculations (primary endpoint) was never analyzed in the study.

Using the data provided by the authors, we know that 20\% in the intervention group developed a MADRS impairment of $\ddagger 5$ points during follow-up. Consequently, if the authors had performed the analysis using the main outcome, they would have found that the trial detected impairment in the intervention group when compared with the control group. These new results certainly do contradict the main conclusions of the trial.

However, the power calculation presented in the article is not correct. Using the data supplied by the authors, we calculated that in order to reach the statistical power assumed by the authors, each study group would have needed 205 patients. A posteriori, however, we could calculate the trial would have been able to detect a difference between the control and the intervention group of $30 \%$.

An intriguing aspect, however, is that the responsible physicians restarted SSRI treatment in seven intervention patients within 4-6 weeks of the start of the trial without any MADRS assessment at this time. In five of those seven patients the reason for restarting SSRI treatment was increased sadness. The outcome of these patients was, however, not considered as "impairment" or "failures". Rather, these patients were evaluated by intention to treat as intervention patients. Following the more appropriate "least favorable principle", one could reasonably assume that at least five patients were actually end-points. Therefore, even using the correct power estimation, the trial would have been able to detect a significant impairment in the intervention group compared with the control group.

In any case, the intention to treat approach is inappropriate in equivalence studies when the underlying hypothesis of the study was that SSRI withdrawal does not alter the risk of depression in the intervention group compared with the control group. That is, control and intervention are equivalent. To realize this aspect we could figure out the worse scenario, where all patients in the intervention group could have restarted SSRI treatment and the authors would have concluded that SSRI withdrawal does not increase the risk of depression.

\section{Inappropriate end-point evaluation}

The same nurse — and also the first author of the arti-cle_-performed all the patient interviews, and she was fully aware of which group (intervention or control) the patient belonged to. The 
same nurse also scored the Health Index, the Symptom Assessment Form, and the Symptoms of Side Effects of SSRI Drug Treatment. The scoring of the MADRS and the Global Assessment of Functioning was done by two psychiatrists, but it is unclear whether this evaluation was completely blind or based on the non-blind interviews recorded by the nurse.

\section{Inappropriate analysis}

We would also like to add some comments to the analysis performed in the trial even if the flaws existing in the conducted experiment make it unacceptable even before any analysis is performed.

The analyses performed by the authors consisted of comparing unadjusted changes in continuous score values between control and intervention for which the trial was not designed and which also conveys severe risk for regression to the mean. Finally, the analysis does not seem appropriate in the presence of unbalanced data. The authors performed a two-factor analysis of variance to adjust but only for the unbalanced gender distribution, which is clearly insufficient. The analysis should include baseline data as covariate. Table 3 did not indicate confidence intervals for differences between the intervention group and the control group and, also, its reading suggests inconsistencies in the computation of confidence intervals and/or $\mathrm{P}$ values for testing between groups.

\section{Summary}

This is an open, non-placebo controlled clinical trial that has been unclearly randomized. We do not know how the randomization was performed, and there is no information on how trial and intervention patients were distributed within the institutions. The statistical power calculation was incorrect and performed for an outcome that was, amazingly, never analyzed. The trial adopted an intention to treat approach that is clearly incorrect since the authors aimed to show equivalence of the effects between the control and the intervention groups.

The endpoint evaluation was open and performed by the first author of the article, which is obviously an inappropriate strategy to ensure the validity of the outcome. The analyses in Table 3 need be clarified.

We would like to end this commentary by referring the statistical principles for clinical trials edited by the European Agency for the Evaluation of Medical Products (EMEA): “The equivalence (or non-inferiority) trial is not conservative in nature, so that many flaws in the design or conduct of the trial will tend to bias the results

towards a conclusion of equivalence. For these reasons, the design features of such trials should receive special attention and their conduct needs special care'” [4]. 
If possible, the trial should be reanalyzed indicating in detail which conclusions can or cannot be stated.

\section{References}

1. Fastbom JBC, Schmidt I, Nordman Ch, Jallow A, Wahlströ m R, Lieberman-Ram H, Styrborn K (2003) Indikatorer för utvärdering av kvaliteten i äldres läkemedelsterapi. Kunskapsöversikt. Swedish National Board of Health and Welfare, Stockholm

2. Fastbom JBC, Olssob J,Olme B, Carlsten A, Schmidt I, Oke T (2004) Kvaliteten i äldres läkemedelsanvändning—KALLA projektet. Lägesbeskrivningar

3. Ulfvarson J, Adami J, Wredling R, Kjellman B, Reilly M, von Bahr C (2003) Controlled withdrawal of selective serotonin reuptake inhibitor drugs in elderly patients in nursing homes with no indication of depression. Eur J Clin Pharmacol 59:735-40.

4. ICH Topic E 9 Statistical principles for clinical trials. The European Agency for the Evaluation of Medical Products). London, 18 March 1998 CPMP/ICH/363/96 